\title{
Smokers' use of electronic cigarettes before, during, and in the month after hospitalization. Findings from the Helping HAND 2 study.
}

\author{
Aleksandra A. Herbec*, MSc, ${ }^{1,2}$, Yuchiao Chang, PhD, ${ }^{1,3}$ Hilary A. Tindle, $\mathrm{MD}^{4}$, and \\ Nancy A. Rigotti, MD ${ }^{1 ., 3}$ \\ ${ }^{1}$ Tobacco Research and Treatment Center, Massachusetts General Hospital, Boston, MA, US; \\ ${ }^{2}$ Department of Behavioral Science and Health, University College London, London, UK; \\ ${ }^{3}$ Division of General Internal Medicine, Department of Medicine, Massachusetts General Hospital and \\ Harvard Medical School, Boston, MA, US; \\ ${ }^{4}$ Vanderbilt Center for Tobacco, Addiction, and Lifestyle (ViTAL), Division of General Internal \\ Medicine and Public Health, Vanderbilt University Medical Center and Vanderbilt Medical School, \\ Nashville, TN, US
}

*Corresponding Author: Aleksandra Herbec, MA (Hons) MSc

Research Department of Behavioural Science and Health

University College London

1-19 Torrington Place,

London, WC1E 6BT, UK

aleksandra.herbec.11@ucl.ac.uk

aaherbec@gmail.com 


\begin{abstract}
Introduction: Hospitalization can motivate smokers to attempt cessation, and some smokers might use e-cigarettes to aid quitting after discharge. Little is known about the patterns and predictors of smokers' e-cigarette use immediately before, during, and after hospitalization.

Methods: Secondary data analysis of a multi-site randomized controlled trial that enrolled 1357 hospitalized smokers planning to quit offered two intensities of post-discharge cessation treatment and reached 1100 participants at 1-month follow-up. We assessed reasons for and patterns of e-cigarette use before, during and immediately after hospitalization. Multivariable logistic regression models identified independent predictors of e-cigarette use.
\end{abstract}

Results: E-cigarettes were used, primarily to aid quitting, by $21.4 \%$ of smokers in the month before admission, $3.1 \%$ during hospitalization, and $18.3 \%$ in the month after discharge. At 1 month, $10.6 \%$ were past 7-day (current) e-cigarette users, but few used e-cigarettes regularly and $57.3 \%$ also smoked tobacco. New e-cigarette use in the month after discharge was rare. The adjusted odds ratio (AOR;95\%CI) for current e-cigarette use was higher among smokers who used e-cigarettes before hospitalization (5.08;3.31-7.81), consumed more alcohol $(1.80 ; 1.16-2.80)$ and had less confidence to quit $(1.66 ; 1.05-2.61)$. The AOR was lower among non-Hispanic blacks $(0.25 ; 0.09-0.71)$, Medicaid-insured $(0.56 ; 0.33-0.93)$, and those randomly assigned to receive free cessation medication and counselling at discharge $(0.58 ; 0.38-0.90)$.

Conclusions: Following hospitalization, some smokers who plan to quit use e-cigarettes, particularly if they used them before admission, are less confident to quit, or have less easy access to conventional treatment. Clinicians should document and be prepared to address ecigarette use among hospitalized smokers.

Keywords: electronic cigarettes; hospitalization; patients; discharge; predictors of use; smoking 


\section{INTRODUCTION}

Electronic cigarettes (e-cigarettes) are battery-operated nicotine delivery devices that heat a nicotine-containing liquid, producing an aerosol that users inhale (Hajek, Etter et al. 2014). Since their introduction in the U.S. and Europe in 2006, e-cigarette use has increased sharply in the general population (Filippidis, Laverty et al. 2017, Zhu, Zhuang et al. 2017). Although e-cigarette use is not risk-free and the long-term health risks are unknown, they may aid cessation (National Academies of Sciences 2018), although evidence from double-blind RCTs to demonstrate this is limited. In the general population of smokers, e-cigarette use is associated with younger age, higher education, White ethnicity, more nicotine dependence and greater interest in quitting, defined as making a recent quit attempt and being a recent exsmoker (Beard, Brose et al. 2013, Brown, West et al. 2014, Biener and Hargraves 2015, Delnevo, Giovenco et al. 2016).

E-cigarette use is relatively common among U.S. adults with tobacco-attributable chronic diseases, who may sometimes require hospitalization (Kruse, Kalkhoran et al. 2017). Ecigarette use in the 30 days before admission increased from $11 \%$ in 2010 to $18.4 \%$ in 2013 in nine U.S. hospitals, coincident with the increase in the general population (Rigotti, Harrington et al. 2015). E-cigarette use in that study was associated with younger age, White race, nonHispanic ethnicity, more education, and greater nicotine dependence (Rigotti, Harrington et al. 2015). A substantial prevalence of e-cigarette use prior to hospitalization (20\%) was also observed among hospitalized patients in the UK in 2012, where it was positively associated with being younger, married or partnered, more educated and exposed to e-cigarette advertisement (Harrington, Hull et al. 2014).

Hospitalization is a health event that motivates many smokers to attempt cessation. The early post-discharge period is a critical time when smokers are vulnerable to resuming smoking, and they might use e-cigarettes to maintain abstinence from combustible tobacco. However, little is known about e-cigarette use in the hospital or soon after discharge (e.g. (Harrington, Hull et al. 2014, Harrington, Cheong et al. 2015, Rigotti, Chang et al. 2018)). A better understanding of smokers' patterns and predictors of e-cigarette use in this context could inform future interventions and clinical guidelines for this population.

This study describes the pattern of e-cigarette use before, during, and immediately after a hospital admission and characterizes independent predictors of e-cigarette use in the month before and after discharge. It analyzed data from the Helping HAND 2 (HH2) randomized controlled trial, which compared the effectiveness of a post-discharge treatment recommendation vs. free comprehensive cessation treatment intervention (Rigotti, Tindle et al. 2016). E-cigarette use was not recommended, but participants' use of e-cigarettes before, during, and after the hospitalization was assessed.

In a separate secondary analysis of $\mathrm{HH} 2$ trial data, e-cigarette use in the 3 months after discharge was associated with less combustible tobacco abstinence at 6 months (Rigotti, Chang et al. 2018). E-cigarette use in the 3 months after discharge was also more common among smokers randomized to receive less easy access to comprehensive tobacco cessation treatment. However, that study did not examine e-cigarette use during hospitalization and immediately post-discharge in detail, nor did it assess factors independently associated with ecigarette use. For the current study, we hypothesized that predictors of use of e-cigarettes 
before hospitalization would resemble those found in the general population, as reviewed above. The analysis of e-cigarette use immediately post-discharge was exploratory, but we expected that e-cigarette use post-discharge would be associated with prior e-cigarette use and with having less easy access to cessation aids post-discharge.

\section{MATERIALS AND METHODS}

\subsection{Design}

Secondary analysis of data from the $\mathrm{HH} 2$ randomized controlled trial that enrolled 1357 cigarette smokers admitted to three hospitals: Massachusetts General Hospital (Boston, Massachusetts), North Shore Medical Center (Salem, MA), and the University of Pittsburgh Medical Center (Pittsburgh, PA) between December 2012 and July 2014. The study was approved by the Institutional Review Board of Partners HealthCare, Inc., and registered with the NIH Clinical Trials Registry (NCT01714323). Details of the HH2 methods (Reid, Regan et al. 2015) and main outcomes (Rigotti, Tindle et al. 2016) have been published. In short, all participants meeting criteria for the $\mathrm{HH} 2$ study (adult, past 30-day smoker smoking $\geq 1$ cigarette daily when smoking normally in the past 30 days, plan to quit or try to quit after hospital discharge) were offered brief smoking cessation counseling and FDA-approved medication in the hospital to manage nicotine withdrawal. At discharge, they were randomly assigned to one of the two conditions: Standard Care or Sustained Care. Standard Care provided a passive referral to the free state quitline and a recommendation for a specific cessation medication. Sustained Care provided up to 90 days of free FDA-approved cessation medication post-discharge (primarily nicotine replacement products) as well as support delivered by automated telephone calls that facilitated medication refills and, upon request, connected smokers directly to a free telephone quitline. E-cigarette use was assessed at enrollment and follow-up, but the intervention protocols provided no advice or guidance about e-cigarettes. Among participants enrolled in the HH2 Study, 33.7\% had a discharge diagnosis from a smoking-related diseases (SRD), including neoplasms, cardiovascular disease, respiratory diseases, and perinatal conditions. Circulatory disease, including cardiovascular, peripheral vascular, and cerebrovascular diseases, was the largest single category, responsible for; $29.3 \%$ of discharge diagnoses (Rigotti et al, 2016).

\subsection{Participants}

This analysis included all participants providing data at baseline $(\mathrm{n}=1357)$ and all participants who were reached for the 1-month follow-up assessment $(n=1100,81 \%)$. To minimize misclassification of e-cigarette use in the analyses of predictors of e-cigarette use at followup, we excluded participants who were not reached at 1-month follow-up or had e-cigarette use missing.

\subsection{Measures}




\subsubsection{Patient baseline characteristics}

Characteristics assessed at baseline included socio-demographic factors (age, sex, ethnicity, education, marital status); years smoking, tobacco dependence (the number of cigarettes per day and time to first cigarette, combined into Heaviness of Smoking Index, HSI, range: 0-6) (Etter, Duc et al. 1999); prior cessation experience (ever tried to quit, ever used cessation medications or behavioral support); confidence to quit (0-10 scale); depression and anxiety symptoms (PHQ-4 score, range: 0-12 (Lowe, Wahl et al. 2010)); smoking related disease (SRD), alcohol consumption (AUDIT-C score, range: 0-12 (Bush, Kivlahan et al. 1998)); use of any illicit drugs in the past year; and health insurance (Medicaid/Medicare/ commercial/other; dichotomized in the regression models into Medicaid vs other).

\subsubsection{E-cigarette use before, during and after hospitalization}

At baseline, all participants were asked about e-cigarettes use (i) in the 30 days prior to hospitalization, and data on (ii) ever use was available for a subsample (see 2.3.3). At 1month follow-up data were collected on (iii) use of e-cigarettes during hospitalization and (iv) where participants used them (indoors, outdoors, or both); (v) use of e-cigarettes since hospitalization, (vi) use of e-cigarettes in the past 30 days, and (vii) use in the past 7 days (a proxy for current use). In logistic regressions, (i) and (vii) were outcomes of interest.

\subsubsection{Additional questions on patterns and reasons for e-cigarette use}

In response to reports of rising e-cigarette use that appeared during study enrollment (CDC), we added additional questions about e-cigarette use to the baseline and follow-up assessments in September 2013. The additional questions asked about ever use of e-cigarettes before hospitalization, frequency of e-cigarette use (the number of days that e-cigarettes were used in the past 30 or 7 days), and the main reasons for use. Response options for the main reason for e-cigarette use at baseline and 1-month follow-up included: Help me to quit smoking cigarettes, Give me something to use in non-smoking area, Use a less risky product than cigarettes long term, or answer Other that invited participants to elaborate. Two researchers analyzed the open-ended responses to Other, identified and coded arising themes with good agreement (Cohen's kappa=.97), and the results were integrated with the closed answer options in the analysis. The expanded surveys were administered to 726 participants at baseline. There were no significant differences in age, sex, race, education level, and nicotine dependence (HSI) between participants who were administered the original vs. the expanded baseline assessment.

\subsubsection{Smoking and cessation behavior after hospitalization}

At 1-month follow-up participants were asked if they had smoked conventional cigarettes or used any other conventional tobacco product (grouped together into 'combustible tobacco') (i) at any time after discharge, (ii) in the past 30 days, and (iii) in the past 7 days (a proxy for smoking currently). We assessed whether (iv) participants relapsed into using combustible tobacco within 7 days after discharge. Participants were also asked about (v) use of cessation 
medication during hospitalization and after discharge, and (vi) use of use of behavioral cessation support after discharge.

\subsection{Analyses}

Data were analyzed using SAS version 9.4 (SAS Institute, Cary NC). Characteristics of participants were compared using Chi-square tests for categorical variables and t-tests for continuous variables between patients who did or did not complete 1-month follow-up assessment, and patients who used and did not use e-cigarettes before, during, and after hospitalization. To identify factors independently associated with e-cigarette use in the 30 days before hospitalization and at 1-month post-discharge, we included all variables that were associated with e-cigarette use at $\mathrm{p}<0.10$ level from the unadjusted analysis. From the model of e-cigarette use post-discharge we excluded e-cigarette use in hospital due to small number of cases and smoking in the past 7 days due to strong correlation with relapse into smoking within 7 days. In sensitivity analyses we investigated models that included e-cigarette use in hospital, or exchanging relapse into smoking within 7 days with smoking in past 7 days. A two-sided p-value of 0.05 or less was considered as statistically significant. Missing data ( $<4.5 \%$ per variable) were not imputed, and were excluded from analyses on a case-by-case basis.

\section{RESULTS}

\subsection{Participants}

A total of 1357 adult smokers participated in the HH2 trial and 1100 completed the 1-month follow-up assessment. Participants who were lost to follow-up were younger (46.1 vs. 50.6 years, $\mathrm{p}<.001)$, smoked cigarettes for fewer years (27.3 vs. 30.9 years, $\mathrm{p}<0.001)$, had higher AUDIT-C scores (3.3 vs. 2.7, $\mathrm{p}=0.014$ ), were less likely to have Medicare (21.4\% vs. $29.9 \%$, $\mathrm{p}=.006$ ), less likely to be diagnosed with smoking-related disease (SRD; $23.7 \%$ vs. $36.1 \%$, $\mathrm{p}<0.001)$, and to have not previously used behavioral cessation support $(10.1 \mathrm{v} .16 .6 \%$, $\mathrm{p}=.009$ ). Among the 1100 who completed the 1-month follow-up assessment, data on ecigarette use was available for 1072 for during the hospital stay, 1096 for e-cigarette use in the past 7 days and 1098 for e-cigarette use in the past 30 days.

\subsection{E-cigarette use before hospitalization}

Past 30-day e-cigarette use was assessed at baseline and reported by $21.4 \%(\mathrm{n}=290)$ of all participants. An additional question on ever use of e-cigarettes was added to the expanded baseline survey, and was answered by 726 participants, among whom $52.8 \%(n=383)$ reported ever use. Baseline characteristics of the 1357 participants according to their use of e-cigarettes in the 30 days prior to hospital admission are presented in Table 1 (for the same data reported as row \% see Table A1 in the Appendix). Patients who used e-cigarettes in the 30 days prior to hospitalization were younger, more likely to be female, had fewer years smoking, and were more likely to have anxiety and depression symptoms. In a multivariable logistic regression 
analysis, younger age (OR $0.77,95 \%$ CI $0.65-0.93$ for every 10 -year increment in age) and being female (OR 1.55, CI 1.18-2.03) were independently associated with e-cigarette use prior to admission.

Table 1: Electronic cigarette use in the 30 days before hospitalization and at 1-month followup: Participant baseline characteristics.

\begin{tabular}{|c|c|c|c|c|c|c|}
\hline \multirow[b]{3}{*}{ Characteristics assessed at baseline } & \multirow{2}{*}{\multicolumn{3}{|c|}{$\begin{array}{c}\begin{array}{c}\text { Sample at baseline } \\
(n=1357)\end{array} \\
\begin{array}{c}\text { E-cigs used } 30 \text { days prior to } \\
\text { hospitalization }\end{array}\end{array}$}} & \multirow{2}{*}{\multicolumn{3}{|c|}{$\begin{array}{c}\text { Sample at 1-month follow-up } \\
\text { (n=1096) } \\
\begin{array}{c}\text { E-cigs used in past } 7 \text { days } \\
\text { (current use) }\end{array}\end{array}$}} \\
\hline & & & & & & \\
\hline & $\begin{array}{c}\text { No } \\
n=1067\end{array}$ & $\begin{array}{c}\text { Yes } \\
\mathbf{n}=290\end{array}$ & $p$ & $\begin{array}{c}\text { No } \\
n=979\end{array}$ & $\begin{array}{c}\text { Yes } \\
n=117\end{array}$ & $p$ \\
\hline Socio-demographic factors & \multicolumn{3}{|c|}{$\%$ column } & \multicolumn{3}{|c|}{$\%$ column } \\
\hline Age, Mean (SD) & $50.3(12.5)$ & $47.4(13.0)$ & $<.001$ & $50.7(12.6)$ & $50.0(11.5)$ & .53 \\
\hline Female, \% & 46.6 & 59.0 & $<.001$ & 48.5 & 59.8 & .021 \\
\hline $\begin{array}{l}\text { Race/ethnicity, \% } \\
\text { White non-Hispanic } \\
\text { Black non-Hispanic } \\
\text { Hispanic } \\
\text { Other including unknown }\end{array}$ & $\begin{array}{c}71.9 \\
15.0 \\
5.0 \\
8.2\end{array}$ & $\begin{array}{c}74.8 \\
10.7 \\
3.8 \\
10.7\end{array}$ & .13 & $\begin{array}{c}70.6 \\
15.4 \\
4.7 \\
9.3\end{array}$ & $\begin{array}{c}83.8 \\
3.4 \\
6.0 \\
6.8\end{array}$ & .003 \\
\hline At least some college, \% & 49.3 & 52.4 & .35 & 49.7 & 63.2 & .006 \\
\hline $\begin{array}{l}\text { Insurance*, } \%(\mathbf{N}) \\
\text { Medicaid } \\
\text { Medicare } \\
\text { Commercial } \\
\text { Other* }\end{array}$ & $\begin{array}{l}29.1 \\
31.0 \\
27.7 \\
12.3\end{array}$ & $\begin{array}{l}25.5 \\
32.4 \\
27.9 \\
14.1\end{array}$ & .62 & $\begin{array}{l}32.0 \\
29.1 \\
26.7 \\
12.4\end{array}$ & $\begin{array}{l}23.1 \\
36.8 \\
29.9 \\
10.3\end{array}$ & .14 \\
\hline \multicolumn{7}{|l|}{ Smoking behavior } \\
\hline Years smoked, Mean(SD) & $30.6(13.7)$ & $28.7(13.4)$ & .034 & $30.9(13.7)$ & $31.1(13.2)$ & .90 \\
\hline Cigarettes per day, Mean(SD) & $15.9(10.0)$ & $16.2(9.6)$ & .67 & $15.6(9.5)$ & $18.2(11.1)$ & .017 \\
\hline Smoked $\leq 30 \mathrm{~min}$ of waking, $\%$ & 74.3 & 77.2 & .31 & 74.1 & 81.2 & .09 \\
\hline Other tobacco in past 30 days, \% & 9.7 & 12.1 & .25 & 10.2 & 8.5 & .57 \\
\hline Ever tried to quit and lasted $\geq 24 \mathrm{~h}, \%$ & 89.8 & 90.7 & .79 & 90.3 & 89.7 & .85 \\
\hline Ever used cessation medications, \% & 69.4 & 74.1 & .12 & 70.1 & 79.5 & .043 \\
\hline Ever used behavioral support, \% & 14.9 & 17.2 & .33 & 16.6 & 16.2 & .91 \\
\hline Confidence in quitting, Mean (SD) & $3.1(1.0)$ & $3.1(1.0)$ & .24 & $3.1(1.0)$ & $3.0(0.9)$ & .17 \\
\hline \multicolumn{7}{|l|}{ Other substances and comorbidities } \\
\hline Alcohol use (AUDIT-C), Mean (SD) & $2.8(3.2)$ & $2.7(3.2)$ & .60 & $2.7(3.1)$ & $2.2(2.8)$ & .04 \\
\hline Any illicit drugs in past year, \%(N) & 27.2 & 30.7 & .24 & 27.3 & 25.6 & .71 \\
\hline PHQ-4, Mean (SD) & $4.7(3.5)$ & $5.2(3.6)$ & .019 & $4.7(3.5)$ & $4.9(3.4)$ & .53 \\
\hline Smoking-related disease, \% & 34.8 & 30.0 & .13 & 35.5 & 39.3 & .42 \\
\hline \multicolumn{7}{|l|}{ Other } \\
\hline $\begin{array}{l}\text { Site, } \% \\
\text { MGH } \\
\text { NSMC } \\
\text { UPMC }\end{array}$ & $\begin{array}{l}38.7 \\
17.5 \\
43.8\end{array}$ & $\begin{array}{l}39.7 \\
13.8 \\
46.6\end{array}$ & .31 & $\begin{array}{l}39.2 \\
18.3 \\
42.5\end{array}$ & $\begin{array}{l}47.0 \\
10.3 \\
42.7\end{array}$ & .07 \\
\hline $\begin{array}{l}\text { HH2 randomized trial group } \\
\text { Intervention } \\
\text { Control }\end{array}$ & $\begin{array}{l}50.5 \\
49.5 \\
\end{array}$ & $\begin{array}{l}48.6 \\
51.4 \\
\end{array}$ & .57 & $\begin{array}{l}52.2 \\
47.8 \\
\end{array}$ & $\begin{array}{l}39.9 \\
60.7 \\
\end{array}$ & .008 \\
\hline
\end{tabular}

M = mean; MGH= Massachusetts General Hospital (Boston, Massachusetts); NSMC= North Shore Medical

Center (Salem, MA); UPMC, University of Pittsburgh Medical Center (Pittsburgh, PA); AUDIT-C = Alcohol Use Disorders Identification Test-(3 items, range, 0-12). Higher values indicate more alcohol use (Bush, Kivlahan et al. 1998); Confidence in ability to quit = range: $1-10$ (1-not at all confident, 10 -very confident $)$ PHQ-4 = The 'Patient Health Questionnaire-4' to assess depression and anxiety symptoms (range: 0-12; point Likert scale with higher values indicate more symptoms) (Lowe, Wahl et al. 2010); Smoking-related diseases are those specified in the 2014 U.S. Surgeon General's Report. These include neoplasms (ICD-9 codes 140-151, 157, 161, 162, 180, 188, 189, 204-208), cardiovascular diseases (ICD-9 codes: 410-414, 390-398, 415-417, 420-429, 430-438, 440448), respiratory diseases (ICD-9 480-492, 496), and perinatal conditions (ICD-9 765, 769, 798.0). *Insurance:

Other included 52 participants with no medical insurance (3.8\%) among all participants assessed at baseline. 


\subsection{E-cigarette use during hospitalization}

Among the 1072 participants who retrospectively reported at 1-month follow-up about ecigarette use in hospital, 33 participants $(3.1 \%)$ reported having used e-cigarettes during hospitalization. Of these, $30(91 \%)$ reported using e-cigarettes inside the hospital building. Indoor use of e-cigarettes was banned at MGH before the trial and during enrollment at NSMC and UPMC, but rates of indoor use were not significantly different by site. In a bivariate analysis, e-cigarettes during hospitalization were more often used by smokers who had used them before hospitalization $(10.5 \%$ vs. $1 \%, \mathrm{p}<.001)$, had at least some college education $(4.3 \%$ vs. $1.7 \%, \mathrm{p}=0.015)$, greater nicotine dependence (HSI mean 3.4 vs 2.8 , $\mathrm{p}=0.019)$, and had no smoking-related disease ( $4.3 \%$ vs. $1.0 \%, \mathrm{p}=.003)$.

\subsection{E-cigarette use after hospitalization}

At the 1-month follow-up, $18.3 \%(\mathrm{n}=201)$ of participants used e-cigarettes at any point after hospitalization, $16.6 \%(n=182)$ in the past 30 days, and $10.7 \%(n=117)$ in the past 7 days (current use). The majority of e-cigarette users also used combustible tobacco (76.9\% of past 30-day e-cigarette users and $57.3 \%$ of past 7 -day e-cigarette users; Table 2). In the subsample of participants answering the expanded survey $(n=609)$, only $3.8 \%$ of never users used ecigarettes for the first time after hospitalization (vs. $27.9 \%$ among those who ever used ecigarettes, $\mathrm{p}<0.001)$.

Baseline characteristics for the participants who were and were not current (past 7 day) ecigarette users at 1-month follow-up are presented in Table 1 (for the same data reported as row \% see Table A1 in the Appendix). Table 3 presents results of unadjusted and adjusted logistic regression models for this sample. In the multivariable logistic regression analysis, higher AUDIT scores, lower confidence to quit, and use of e-cigarettes before hospitalization were positively associated with current e-cigarette use. Being non-Hispanic black, having Medicaid insurance, and being randomized to receive easier access to cessation support remained significantly and negatively associated with current e-cigarettes use post-discharge. There was also a difference across study sites in current e-cigarette use. The results remained essentially the same in sensitivity analyses.

Table 2: Use of e-cigarettes and combustible tobacco at 1 month after hospital discharge.

\begin{tabular}{|c|c|c|c|}
\hline $\begin{array}{l}\text { E-cigarette use at 1-month } \\
\text { follow-up }\end{array}$ & \multicolumn{2}{|c|}{ Row \% / \% of total sample (N) } & \\
\hline \multirow[t]{2}{*}{$\begin{array}{l}\text { Used e-cigarettes in the past } \\
30 \text { days }\left(\mathrm{n}=1097^{*}\right)\end{array}$} & \multicolumn{2}{|c|}{$\begin{array}{c}\text { Smoked conventional cigarettes in the past } 30 \\
\text { days }\end{array}$} & $\mathbf{p}$ \\
\hline & No & Yes & \\
\hline Yes $(n=182)$ & $23.1 / 3.8(42)$ & $76.9 / 12.8(140)$ & $<0.001$ \\
\hline No $(n=915)$ & $42.2 / 35.2(386)$ & $57.8 / 48.2(529)$ & \\
\hline \multirow{2}{*}{$\begin{array}{l}\text { Used e-cigarettes in the past } 7 \\
\text { days (current use) }\left(\mathrm{n}=1095^{*}\right)\end{array}$} & \multicolumn{2}{|c|}{ Smoked conventional cigarettes in the past 7 days } & \\
\hline & No & Yes & \\
\hline Yes $(n=117)$ & $42.7 / 4.6(50)$ & $57.3 / 6.1(67)$ & $<0.001$ \\
\hline No $(n=978)$ & $53.0 / 47.3(518)$ & $(47.0 / 42.0(460)$ & \\
\hline
\end{tabular}

* 1 participant with missing data 
Table 3: Current (past 7 day) e-cigarette use at 1-month post-discharge: participant characteristics and factors associated with use.

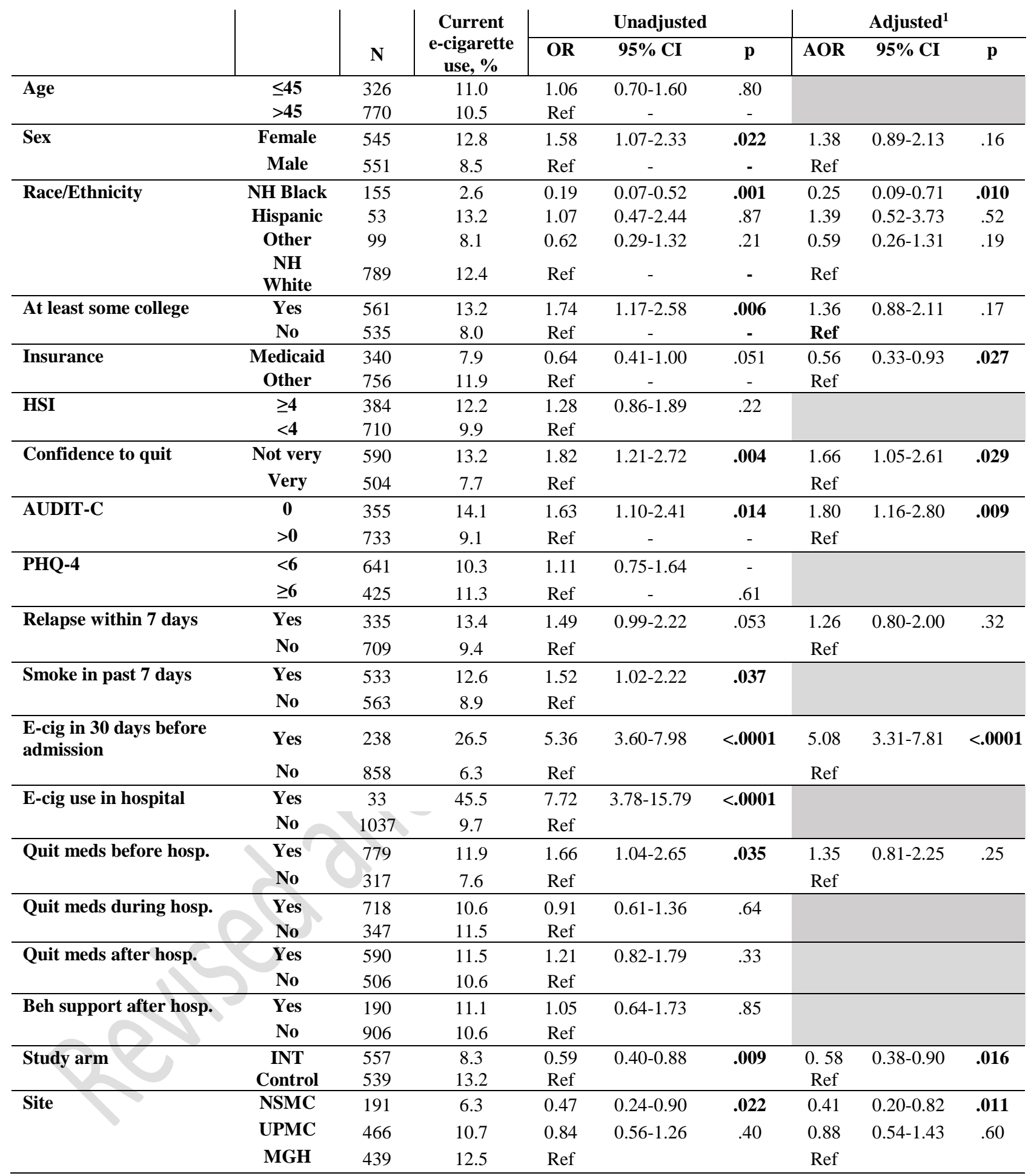

Hosp - hospitalization; NH - non-Hispanic; HSI = Heaviness of Smoking Index combining information on cigarettes per day and time to cigarette rage: $0=$ very low dependence, $6=$ very high dependence); Confidence to quit ( $1=$ not at all; $10=$ very high); PHQ-4= The 'Patient Health Questionnaire-4' assessing depression and anxiety symptoms (4 point Likert scale; higher values indicate more symptoms) (Lowe, Wahl et al. 2010); MGH=Massachusetts General Hospital (Boston, Massachusetts); NSMC=North Shore Medical Center (Salem, MA); UPMC=University of Pittsburgh Medical Center (Pittsburgh, PA); INT - Intervention; ${ }^{1}$ Model adjusted for all predictors that were significant in unadjusted analysis ( $p<0.1$ ), except of: 'smoking cigarettes in the past 7 days' which showed high collinearity with variable 'relapsed to smoking within 7 days of follow-up' but had less predictive power; and variable 'using e-cigarettes during hospitalization', which was significantly related to post-discharge e-cigarette use, but had too few cases ( $\mathrm{n}=33$ ) to include in the fully adjusted model. Variables shaded in grey in the adjusted model columns were not included in the final model. 


\subsection{Frequency and reasons for e-cigarette use before and after hospitalization}

Participants who used e-cigarettes in the 30 days before admission reported having used them on a median of 4 (IQR: 2-10) of 30 days. The most common reason for e-cigarette use in the past 30 days was as an aid to quitting (61.5\%), followed by use in non-smoking areas (12.2\%), experimenting (9\%), use as a less risky product long-term (3.8\%), to cut down on cigarettes $(3.8 \%)$, temporary replacement of cigarettes $(2.6 \%)$, for financial reasons $(1.3 \%)$, and $5.1 \%$ for other reasons.

At one-month follow-up, among participants who reported data on frequency of e-cigarette use, the median number of days of use was $10(\mathrm{IQR}=3-20)$ of the past 30 days $(n=110)$, and 4 $(\mathrm{IQR}=2-7)$ in the past 7 days $(\mathrm{n}=73)$. The majority $(76.0 \%)$ of the participants who provided data on main reasons for e-cigarette use post-discharge used them as aids to quit smoking, $6.6 \%$ as a less risky product, $6.6 \%$ to occupy their hands or mouth, $1.7 \%$ in non-smoking areas, $1.7 \%$ were experimenting, and $0.8 \%$ as temporary replacement of cigarettes, and $0.8 \%$ to cut down.

\section{DISCUSSION}

\subsection{Overview of findings}

This study assessed e-cigarette use and predictors of use before, during and after hospitalization among smokers who were interested in quitting and had enrolled in a comparative effectiveness trial of smoking treatment after hospital discharge (Rigotti, Tindle et al. 2016). One-fifth of hospitalized smokers who planned to quit after discharge reported having used e-cigarettes in the 30 days before hospital admission. Although all participants received smoking cessation assistance in hospital and after discharge, 18\% participants used e-cigarette at any time in the month after discharge, and $11 \%$ were currently using them at the 1 month-follow-up. While e-cigarettes were used primarily to quit, the frequency of use was low, and most e-cigarette users continued using combustible tobacco after discharge. Initiation of e-cigarette use post-discharge with no prior experience of use was very uncommon.

\subsection{E-cigarettes use before and during hospitalization}

The prevalence and factors independently associated with e-cigarette use before hospitalization in this study - younger age and being female - resemble those found in other studies of e-cigarette users (Brown, West et al. 2014, Cummins, Zhu et al. 2014, Harrington, Hull et al. 2014). Although participants were more ill than the general population, they might not have expected or planned to be hospitalized and thus their e-cigarette use reflected patterns seen among adult smokers in general. Use of e-cigarettes during hospitalization was rare, and the small sample precluded conducting an adjusted analysis, but there was an indication that those with prior use of e-cigarettes and greater dependence are more likely to use them. Nevertheless, as bans on e-cigarettes use in hospitals become established. this behavior may become even less frequent. 


\subsection{E-cigarette use in the month after discharge}

With regards to e-cigarette use after hospitalization, ever use of e-cigarettes was strongly associated with post-discharge use, and e-cigarette use in the 30 days before hospitalizations remained the strongest predictor of use at 1-month post-discharge in the adjusted analysis. Additionally, and echoing other findings from the HH2 study (Rigotti, Chang et al. 2018), participants randomized to the arm offering easier access to conventional tobacco cessation treatment were less likely to report using e-cigarettes after discharge. The easier access to conventional tobacco cessation treatment in one study arm may partly explain the lower prevalence of e-cigarette use after hospitalization in comparison to before hospitalization. Among other independent and positive predictors of e-cigarette use at 1-month after discharge was low confidence to quit and not having Medicaid insurance, the latter acting as a proxy for lower-socio-economic status (Foraker, Rose et al. 2010). Past or current use of cessation pharmacotherapy or behavioral support were not related to e-cigarette use post-discharge.

Taken together, this study suggests that patients who plan to quit but are less confident in their ability to do so, may have higher income, and are not provided with easy access to conventional treatment may choose to use e-cigarettes, particularly if they have prior experience with e-cigarette use. In light of research showing that e-cigarette is associated with poorer cessation outcomes, (Rigotti, Chang et al. 2018), this could be a potential cause for concern. Similarly, in a recent study of primary care patients enrolled in a traditional cessation treatment program (Zawertailo, Pavlov et al. 2016), e-cigarette use during treatment was not only common (18\%), but also associated with lower quit rates at 3-month (27.0\% vs. $36.4 \%)$ and 6-month follow-up (26.3\% vs. $42.0 \%$ ).

Finally, both before and after hospitalization, most participants reported using e-cigarettes primarily as aids to quitting, a finding consistent with prior studies among the general adult population of smokers (Berg, Barr et al. 2014, Brown, West et al. 2014, Rutten, Blake et al. 2015). Notably, there was some indication that the frequency of e-cigarette use increased from pre-hospitalization to post-discharge, and that a greater proportion of e-cigarette users reported using them to quit after hospitalization. One possible explanation is that this reflected participants' greater interest and motivation to quit smoking cigarettes, which could be motivated by hospitalization and illness, or due to enrolling in a stop smoking study, where complete abstinence was encouraged and assessed.

Nevertheless, most participants still used e-cigarettes less than daily, which is associated with less cessation (Coleman, Rostron et al. 2018). Moreover, few participants in the study switched completely to e-cigarettes. This dual pattern of e-cigarette use is associated with a lower likelihood of cessation in general populations (National Academies of Sciences 2018) and may be a special concern for recently-hospitalized smokers, who are at higher risk of adverse health outcomes from continued smoking than is the general population of smokers. Dual users may also perceive that they reduce tobacco-related health risks by intermittently using e-cigarettes, but few data demonstrate that reducing use without stopping all combustible cigarette use reduces mortality or morbidity (Inoue-Choi, Liao et al. 2017) (Hackshaw, Morris et al. 2018). One study of smokers with asthma who used e-cigarettes had improved lung function (Polosa, Morjaria et al. 2014), but more evidence is needed. 


\subsection{Implications}

Clinical guidelines and hospital quality regulations already require the assessment of tobacco use at hospital admission (Fiore 2008, Fiore, Goplerud et al. 2012). The present findings suggest that e-cigarette use should also be assessed routinely among patients, and that clinicians should anticipate that hospitalized smokers who are interested in quitting and have previously used e-cigarettes may continue to use them after discharge, even if FDA-approved medications and cessation support are recommended. The lack of data on the efficacy of ecigarettes as cessation aids makes a clinical recommendation about their use premature, but clinicians can inform patients about this uncertainty, encourage patients to use evidence-based treatments first (Fiore 2008), and if they still decide to use e-cigarettes, emphasize the need to cease using conventional tobacco altogether, as national guidelines have recommended, e.g. the American Heart Association (Bhatnagar, Whitsel et al. 2014) or The American Cancer Society (The American Cancer Society 2018).

Furthermore, although the present study has not assessed such factors, a number of additional reasons could explain why smokers and hospitalized patients use e-cigarettes. These include beliefs about the effectiveness and safety of e-cigarettes, especially in relation to combustible cigarettes (Ambrose, Rostron et al. 2014, Majeed, Weaver et al. 2017), and to FDA-approved medications. These should be investigated to develop more comprehensive interventions.

\subsection{Strength and Limitations}

Strengths of this study include the large cohort assembled from three study sites, a more detailed assessment of e-cigarette use that previously published, and the novel assessment of e-cigarette use in the month after hospital discharge. The study had also limitations. First, the findings cannot be generalized to all hospitalized smokers because the sample was limited to those who planned to quit after discharge and enrolled in a randomized trial of a cessation intervention. It had the advantage of being a multi-site study conducted in two U.S. regions and in both community and academic hospitals, but the prevalence and predictors of ecigarette use could be different in other parts of the U.S. or in other types of hospitals (e.g., safety-net hospitals). Additionally, participants who were lost to follow-up were younger and healthier. Because age is associated with e-cigarette use, the overall prevalence of e-cigarettes post-discharge might have been higher if these individuals had been reached.. Another limitation is that questions on ever use, frequency and reasons for e-cigarette use were added midway through enrollment; however, the characteristics of respondents who were asked the full set of questions did not differ from those asked only a subset.

\section{CONCLUSIONS}

Substantial minorities of smokers who planned to quit used e-cigarettes before hospitalization, and many of these continued to use e-cigarettes after discharge, often together with combustible tobacco, and even when conventional smoking cessation therapies were offered or recommended. At the same time, few hospitalized smokers initiated e-cigarette use postdischarge without prior experience of use. Physicians caring for smokers who are currently or 
recently hospitalized should routinely ask about the use of e-cigarettes before hospitalization, be prepared to address the use of e-cigarette post-discharge, and emphasize the need for, and benefit of, complete cessation of combustible tobacco regardless of the cessation aids used. 


\section{REFERENCES}

Ambrose, B. K., B. L. Rostron, S. E. Johnson, D. B. Portnoy, B. J. Apelberg, A. R. Kaufman and C. J. Choiniere (2014). "Perceptions of the relative harm of cigarettes and e-cigarettes among U.S. youth." Am J Prev Med 47(2 Suppl 1): S53-60.

Beard, E., L. S. Brose, J. Brown, R. West and A. McEwen (2013). "How are the English Stop Smoking Services responding to growth in use of electronic cigarettes?" Patient Educ Couns.

Berg, C. J., D. B. Barr, E. Stratton, C. Escoffery and M. Kegler (2014). "Attitudes toward E-Cigarettes, Reasons for Initiating E-Cigarette Use, and Changes in Smoking Behavior after Initiation: A Pilot Longitudinal Study of Regular Cigarette Smokers." Open J Prev Med 4(10): 789-800.

Bhatnagar, A., L. P. Whitsel, K. M. Ribisl, C. Bullen, F. Chaloupka, M. R. Piano, R. M. Robertson, T. McAuley, D. Goff, N. Benowitz, C. o. C. American Heart Association Advocacy Coordinating Committee, C. o. C. C. Stroke Nursing, C. Council on Quality of and R. Outcomes (2014). "Electronic cigarettes: a policy statement from the American Heart Association." Circulation 130(16): 1418-1436.

Biener, L. and J. L. Hargraves (2015). "A longitudinal study of electronic cigarette use among a population-based sample of adult smokers: association with smoking cessation and motivation to quit." Nicotine Tob Res 17(2): 127-133.

Brown, J., R. West, E. Beard, S. Michie, L. Shahab and A. McNeill (2014). "Prevalence and characteristics of e-cigarette users in Great Britain: Findings from a general population survey of smokers." Addict Behav 39(6): 1120-1125.

Bush, K., D. R. Kivlahan, M. B. McDonell, S. D. Fihn and K. A. Bradley (1998). "The AUDIT alcohol consumption questions (AUDIT-C): an effective brief screening test for problem drinking. Ambulatory Care Quality Improvement Project (ACQUIP). Alcohol Use Disorders Identification Test." Arch Intern Med 158(16): 1789-1795.

CDC (2013). Notes from the field: electronic cigarette use among middle and high school studentsUnited States, 2011-2012. MMWR Morb Mortal Wkly Rep20136272930.

Coleman, B., B. Rostron, S. E. Johnson, A. Persoskie, J. Pearson, C. Stanton, K. Choi, G. Anic, M. L. Goniewicz, K. M. Cummings, K. A. Kasza, M. L. Silveira, C. Delnevo, R. Niaura, D. B. Abrams, H. L. Kimmel, N. Borek, W. M. Compton and A. Hyland (2018). "Transitions in electronic cigarette use among adults in the Population Assessment of Tobacco and Health (PATH) Study, Waves 1 and 2 (2013-2015)." Tobacco Control: tobaccocontrol-2017-054174.

Cummins, S. E., S. H. Zhu, G. J. Tedeschi, A. C. Gamst and M. G. Myers (2014). "Use of e-cigarettes by individuals with mental health conditions." Tob Control 23 Suppl 3: iii48-53.

Delnevo, C. D., D. P. Giovenco, M. B. Steinberg, A. C. Villanti, J. L. Pearson, R. S. Niaura and D. B. Abrams (2016). "Patterns of Electronic Cigarette Use Among Adults in the United States." Nicotine Tob Res 18(5): 715-719.

Etter, J. F., T. V. Duc and T. V. Perneger (1999). "Validity of the Fagerstrom test for nicotine dependence and of the Heaviness of Smoking Index among relatively light smokers." Addiction 94(2): 269-281.

Filippidis, F. T., A. A. Laverty, V. Gerovasili and C. I. Vardavas (2017). "Two-year trends and predictors of e-cigarette use in 27 European Union member states." Tob Control 26(1): 98-104.

Fiore, M. (2008). "Treating tobacco use and dependence: 2008 update: Clinical practice guideline.".

Fiore, M. C., E. Goplerud and S. A. Schroeder (2012). "The Joint Commission's new tobacco-cessation measures--will hospitals do the right thing?" N Engl J Med 366(13): 1172-1174.

Foraker, R. E., K. M. Rose, E. A. Whitsel, C. M. Suchindran, J. L. Wood and W. D. Rosamond (2010). "Neighborhood socioeconomic status, Medicaid coverage and medical management of myocardial infarction: atherosclerosis risk in communities (ARIC) community surveillance." BMC Public Health 10: 632.

Hackshaw, A., J. K. Morris, S. Boniface, J. L. Tang and D. Milenkovic (2018). "Low cigarette consumption and risk of coronary heart disease and stroke: meta-analysis of 141 cohort studies in 55 study reports." BMJ 360: j5855.

Hajek, P., J. F. Etter, N. Benowitz, T. Eissenberg and H. McRobbie (2014). "Electronic cigarettes: review of use, content, safety, effects on smokers and potential for harm and benefit." Addiction 109(11): 1801-1810. 
Harrington, K., J. Cheong, S. Hendricks, C. Kohler and W. Bailey (2015). "E-cigarette and Traditional Cigarette Use Among Smokers During Hospitalization and 6 Months Later." Cancer Epidemiology Biomarkers \& Prevention 24(4): 762-762.

Harrington, K. F., N. C. Hull, O. Akindoju, Y. I. Kim, P. S. Hendricks, C. Kohler and W. C. Bailey (2014). "Electronic cigarette awareness, use history, and expected future use among hospitalized cigarette smokers." Nicotine Tob Res 16(11): 1512-1517.

Inoue-Choi, M., L. M. Liao, C. Reyes-Guzman, P. Hartge, N. Caporaso and N. D. Freedman (2017). "Association of Long-term, Low-Intensity Smoking With All-Cause and Cause-Specific Mortality in the National Institutes of Health-AARP Diet and Health Study." JAMA Intern Med 177(1): 8795.

Kruse, G. R., S. Kalkhoran and N. A. Rigotti (2017). "Use of Electronic Cigarettes Among U.S. Adults With Medical Comorbidities." Am J Prev Med 52(6): 798-804.

Lowe, B., I. Wahl, M. Rose, C. Spitzer, H. Glaesmer, K. Wingenfeld, A. Schneider and E. Brahler (2010). "A 4-item measure of depression and anxiety: validation and standardization of the Patient Health Questionnaire-4 (PHQ-4) in the general population." J Affect Disord 122(1-2): 86-95.

Majeed, B. A., S. R. Weaver, K. R. Gregory, C. F. Whitney, P. Slovic, T. F. Pechacek and M. P. Eriksen (2017). "Changing Perceptions of Harm of E-Cigarettes Among U.S. Adults, 2012-2015." Am J Prev Med 52(3): 331-338.

National Academies of Sciences, E., and Medicine (2018). Public health consequences of e-cigarettes. Washington, DC, The National Academies Press.

Polosa, R., J. Morjaria, P. Caponnetto, M. Caruso, S. Strano, E. Battaglia and C. Russo (2014). "Effect of smoking abstinence and reduction in asthmatic smokers switching to electronic cigarettes: evidence for harm reversal." Int J Environ Res Public Health 11(5): 4965-4977.

Reid, Z. Z., S. Regan, J. H. Kelley, J. M. Streck, T. Ylioja, H. A. Tindle, Y. Chang, D. E. Levy, E. R. Park, D. E. Singer, K. M. Carpenter, M. Reyen and N. A. Rigotti (2015). "Comparative Effectiveness of Post-Discharge Strategies for Hospitalized Smokers: study protocol for the Helping HAND 2 randomized controlled trial." BMC Public Health 15: 109.

Rigotti, N. A., Y. Chang, H. A. Tindle and et al. (2018). "Association of e-cigarette use with smoking cessation among smokers who plan to quit after a hospitalization: A prospective study." Annals of Internal Medicine.

Rigotti, N. A., K. F. Harrington, K. Richter, J. L. Fellows, S. E. Sherman, E. Grossman, Y. Chang, H. A. Tindle, T. Ylioja and T. Consortium of Hospitals Advancing Research on (2015). "Increasing prevalence of electronic cigarette use among smokers hospitalized in 5 US cities, 2010-2013." Nicotine Tob Res 17(2): 236-244.

Rigotti, N. A., H. A. Tindle, S. Regan, D. E. Levy, Y. Chang, K. M. Carpenter, E. R. Park, J. H. Kelley, J. M. Streck, Z. Z. Reid, T. Ylioja, M. Reyen and D. E. Singer (2016). "A Post-Discharge SmokingCessation Intervention for Hospital Patients: Helping Hand 2 Randomized Clinical Trial." Am J Prev Med 51(4): 597-608.

Rutten, L. J., K. D. Blake, A. A. Agunwamba, R. A. Grana, P. M. Wilson, J. O. Ebbert, J. Okamoto and S. J. Leischow (2015). "Use of E-Cigarettes Among Current Smokers: Associations Among Reasons for Use, Quit Intentions, and Current Tobacco Use." Nicotine Tob Res 17(10): 1228-1234.

The American Cancer Society, I. (2018). American Cancer Society Position Statement on Electronic Cigarettes.

Zawertailo, L., D. Pavlov, A. Ivanova, G. Ng, D. Baliunas and P. Selby (2016). "Concurrent E-Cigarette Use During Tobacco Dependence Treatment in Primary Care Settings: Association With Smoking Cessation at Three and Six Months." Nicotine Tob Res.

Zhu, S. H., Y. L. Zhuang, S. Wong, S. E. Cummins and G. J. Tedeschi (2017). "E-cigarette use and associated changes in population smoking cessation: evidence from US current population surveys." BMJ 358: j3262. 